religion into routine psychiatric practice in the UK. Nonetheless, there is growing controversy on the subject. We believe that a number of statements, including the previous president's apparent support for Koenig's proposals (e.g. praying with patients or consultation with clergy) create a real and undesirable ambiguity as to the limits of generally acceptable clinical practice with respect to religion and spirituality. In a paper presently in press, ${ }^{5}$ we argue that Koenig's proposals are in breach of General Medical Council guidance. It would be unrealistic to expect to resolve all of the current issues of dispute in the immediate future, but we would suggest that it would be possible to identify the boundaries of acceptable clinical practice with regard to the points of greatest controversy.

In 2006, the American Psychiatric Association published guidance on 'religious/spiritual commitments and psychiatric practice' (www.psych.org/Departments/EDU/Library/ APAOfficialDocumentsandRelated/ResourceDocuments/ 200604.aspx). It would be timely for the Royal College of Psychiatrists to develop similar guidance. We call on the president to establish a working group to produce guidelines on broad principles and, in addition, to address a narrow range of specific issues.

- Is it acceptable to pray with patients? If so, under what circumstances and with what safeguards?

- Should a spiritual history be taken from all patients? Should this include atheists?

- Is it acceptable for psychiatrists to challenge unhealthy religious beliefs? How can this be assessed reliably? How can it be distinguished from proselytising?

- Should members of the College who write scientific papers for journals concerning religion or spirituality declare their religious aliation as a conflict of interest?

Given the depth of feeling expressed in recent correspondence, the task may appear daunting. However, this subject demands serious and immediate attention exactly because it is difficult and contentious. A carefully composed and wellchaired working group that had credibility with all shades of opinion could produce guidance that would allow us to move on from simply restating our disagreements. It would allow service users to know what to expect when they consult us.

Declaration of interest

R.P. is an atheist. R.H. is a Buddhist.

1 Cook $\mathrm{CCH}$, Dein S, Powell A, Eagger S. Research in spirituality and mental health. Psychiatrist 2010; 34: 304.

2 Koenig HG. Handbook of Religion and Mental Health. Academic Press, 1998.

3 Sloan RP. Blind Faith: The Unholy Alliance of Religion and Medicine. St Martin's Griffin, 2008.

4 Sloan R, Bagiella E, Powell T. Religion, spirituality and medicine. Lancet 1999; 353: 664-7.

5 Poole R, Higgo R. Spirituality and the threat to therapeutic boundaries in psychiatric practice. Ment Health Relig Cult 2010, in press.

Rob Poole, Professor of Mental Health, Glyndwr University, Wrexham, Wales, email: rob.poole@wales.nhs.uk, Robert Higgo, Consultant Psychiatrist, Liverpool Assertive Outreach Team, Liverpool. doi: 10.1192/pb.34.10.452b

\section{Inexperienced trainees doing more Section 136 emergency assessments}

Opportunities for emergency assessments by junior trainees are certainly being reduced, largely as a result of rota merges to comply with the European Working Time Directive for doctors ${ }^{1}$ and New Deal. ${ }^{2}$ However, rather paradoxically, in areas where Section 136 suites have been created as an alternative to police custody, there is now often an expectation that such assessments are undertaken by these same juniors who have little experience of risk assessments and management of acute psychiatric presentations. When similarly detained patients are taken to police custody they automatically see the senior, Section 12-approved doctor on call.

Although the Mental Health Act Code of Practice states that the doctor examining a patient detained under Section 136 should 'wherever possible be approved under Section 12 of the Act', considerable national variation exists in the interpretation of this statement. Therefore, patients detained under Section 136 who are brought to a Section 136 suite are frequently assessed by a junior doctor with minimal (and ever reducing) experience of acute psychiatry or the Mental Health Act, potentially even doing their first ever on-call in the specialty. Training around the Mental Health Act is patchy, supervision is often poor and documentation of these assessments is variable.

Although the Code of Practice suggests that the examining doctor should discuss the patient with both the approved mental health professional and senior doctor on call, for a variety of reasons this does not always happen and the Code is clear that the decision is that of the assessing doctor and not that of the Section 12 doctor. Even where the senior doctor is consulted by telephone, they will base their advice on the information presented by the junior trainee.

In addition, the Code states clearly that where the assessing doctor fails to detect any form of mental disorder the person should be discharged from detention immediately, with no requirement to be seen by the approved mental health professional. So these inexperienced junior doctors are doing complex assessments typically out of hours, often with limited support and training and times taking sole responsibility for discharging patients.

Ideally, trainees in the first few months of their psychiatry rotation should not be undertaking Section 136 assessments at all. With good supervision, a clear policy and adequate training it may be appropriate for juniors with more experience to do these assessments within a hospital setting but senior input should be expected. Patients detained under Section 136 deserve to be seen in an appropriate environment, which, wherever possible, should not be police custody, but above all they deserve a robust assessment by an appropriately experienced psychiatrist.

1 Waddell L, Crawford C. Junior doctors are performing fewer emergency assessments - a cause for concern. Psychiatrist 2010; 34: 268-70.

2 Department of Health. Reducing Junior Doctors' Hours Continuing Action to Meet New Deal Standards Rest Periods and Working Arrangements, Improving Catering and Accommodation for Juniors, Other Action Points (HSC 1998/240). Department of Health, 1998.

Liz Tate, Specialty Registrar, Forensic Psychiatry, Ravenswood House Medium Secure Unit, Fareham, Hampshire, email: liztate@doctors.org.uk doi: $10.1192 / \mathrm{pb} .34 .10 .453$

\section{Junior doctors are performing fewer emergency assessments}

Waddell \& Crawford ${ }^{1}$ have demonstrated very clearly that trainees are becoming more and more limited in their experience of emergency psychiatry. This is, to use their own 